\title{
Identification of partial SLC2OA2 deletions in primary brain calcification using whole-exome sequencing
}

\begin{abstract}
Stéphanie David ${ }^{1,2,16}$, Joana Ferreira ${ }^{1,2,3,4,16}$, Olivier Quenez ${ }^{1,2,5}$, Anne Rovelet-Lecrux ${ }^{1,2}$, Anne-Claire Richard ${ }^{2,5}$, Marc Vérin ${ }^{6}$, Snejana Jurici ${ }^{7}$, Isabelle Le Ber ${ }^{8,9,10}$, Anne Boland ${ }^{11}$, Jean- François Deleuze ${ }^{11}$, Thierry Frebourg ${ }^{1,2,12}$, João Ricardo Mendes de Oliveira ${ }^{3,13}$, Didier Hannequin ${ }^{1,2,5,12,14}$, Dominique Campion ${ }^{1,2,5,15}$ and Gaël Nicolas ${ }^{\star, 1,2,5,12}$

Primary brain calcification (PBC) is a dominantly inherited calcifying disorder of the brain. SLC2OA2 loss-of-function variants account for the majority of families. Only one genomic deletion encompassing SLC2OA2 and six other genes has been reported. We performed whole-exome sequencing (WES) in 24 unrelated French patients with PBC, negatively screened for sequence variant in the known genes SLC2OA2, PDGFB, PDGFRB and XPR1. We used the CANOES tool to detect copy number variations (CNVs). We detected two deletions of exon 2 of SLC2OA2 in two unrelated patients, which segregated with PBC in one family. We then reanalyzed the same series using a QMPSF assay including one amplicon in each exon of SLC20A2 and detected two supplemental partial deletions in two patients: one deletion of exon 4 and one deletion of exons 4 and 5 . These deletions were missed by the first screening step of CANOES but could finally be detected after readjustment of bioinformatic parameters and use of a genotyping step of CANOES. This study reports the first partial deletions of SLC2OA2 and strengthens its position as the major PBC-causative gene. It is possible to detect short CNVs from WES data, although the sensitivity of such tools should be evaluated in comparison with other methods.
\end{abstract}

European Journal of Human Genetics (2016) 24, 1630-1634; doi:10.1038/ejhg.2016.50; published online 1 June 2016

\section{INTRODUCTION}

Primary brain calcification $(\mathrm{PBC})$, also known as primary familial brain calcification (OMIM 213600) or formerly Fahr's disease, is defined by the presence of microvascular calcification affecting at least the basal ganglia with no cause following an extensive clinical, biological, and imaging etiological assessment. Calcification carriers may exhibit diverse neurologic or psychiatric symptoms. Among them, the most frequent three categories are: psychiatric disturbances (mainly mood, psychotic or personality disorders), cognitive impairment (involving mainly memory and executive functions), and movement disorders (mainly extrapyramidal signs). ${ }^{1}$ Patients may present other symptoms such as gait disorder, cerebellar syndrome, dysarthria, and rarely seizures. Cephalalgia and especially migraine are frequent circumstances allowing the identification of brain calcification. ${ }^{1}$

PBC is inherited as an autosomal dominant trait. In this context, causative variants have been identified in 4 genes: SLC20A2 (OMIM 158378), ${ }^{2}$ PDGFRB (OMIM 173410), ${ }^{3}$ PDGFB (OMIM 190040), ${ }^{4}$ and XPR1 (OMIM 605237). ${ }^{5}$ Two copy number variants (CNVs) were previously detected as PBC-causing: a partial deletion of PDGFB in one patien $\mathrm{t}^{6}$ and a deletion encompassing SLC2OA2 entirely and six other genes in one family. ${ }^{7}$ SLC2OA2 and XPR1 encode inorganic phosphate transporters, the importer SLC20A2 (also known as PiT2), and the exporter XPR1, respectively. Loss of function of either gene leads to microvascular mural and perivascular calcification involving vascular smooth muscle cells and pericytes. Loss-of-function variants of PDGFB or PDGFRB are responsible for an alteration of the bloodbrain barrier (BBB). Whether the BBB alteration is the direct cause of microvascular calcification or there is other links with inorganic phosphate metabolism remains to be determined. ${ }^{8}$ In our French series of PBC patients, $>50 \%$ of probands or sporadic cases do not exhibit any causative variant in one of these genes after Sanger sequencing (unpublished data).

We performed whole-exome sequencing (WES) in 24 patients with PBC negatively screened for the four genes. Although WES was developed to detect single nucleotide variants and short indels, we can now take advantage from WES data to detect CNVs. We report here the results of CNV analysis of these four genes in these 24 WES.

\section{MATERIALS AND METHODS}

Patients

Patients were included following the previously described criteria. ${ }^{9}$ In brief, probands exhibited (1) at least bilateral lenticular calcification, (2) the total calcification score using our own visual rating scale was above the age-specific threshold, and (3) an extensive etiological assessment was normal.

${ }^{1}$ Inserm U1079, Rouen University, IRIB, Normandy University, Rouen, France; ${ }^{2}$ Normandy Centre for Genomic Medicine and Personalized Medicine, Rouen, France; ${ }^{3}$ Keizo Asami Laboratory, Federal University of Pernambuco, Recife, Brazil; ${ }^{4}$ Biological Sciences Graduate Program, Federal University of Pernambuco, Recife, Brazil; ${ }^{5} \mathrm{CNR}$-MAJ, Rouen University Hospital, Rouen, France; ' ${ }^{6}$ epartment of Neurology, Pontchaillou Hospital, Rennes University Hospital, Rennes, France; ${ }^{7}$ Department of Neurology, Perpignan Hospital, Perpignan, France; ${ }^{8}$ Sorbonne Universités, UPMC Univ Paris 06, Inserm UMRS1127, CNRS UMR 7225, ICM, Paris, France; ${ }^{9}$ AP-HP, Hôpital de la Pitié-Salpêtrière, Centre de Référence des Démences Rares, Paris, France; ${ }^{10} \mathrm{AP}-\mathrm{HP}$, Département des maladies du système nerveux, Hôpital de la Pitié-Salpêtrière, Paris, France; ${ }^{11}$ Centre National de Génotypage, Institut de Génomique, CEA, Evry, France; ${ }^{12}$ Department of Genetics, Rouen University Hospital, Rouen, France; ${ }^{13}$ Department of Neuropsychiatry, Federal University of Pernambuco, Recife, Brazil; ${ }^{14}$ Department of Neurology, Rouen University Hospital, Rouen, France; ${ }^{15}$ Department of Research, Rouvray Psychiatric Hospital, Sotteville-lès-Rouen, France

*Correspondence: Dr G Nicolas, Inserm U1079, Faculté de médecine, Rouen University, IRIB, Normandy University, 22, Boulevard Gambetta, 76183 Rouen, France. Tel: +33 235148280, Fax: +33 235148237; E-mail: gaelnicolas@hotmail.com

${ }^{16}$ These authors contributed equally to this work.

Received 11 December 2015; revised 12 April 2016; accepted 15 April 2016; published online 1 June 2016 
Genetic analyses were performed after written, informed consent. This study was approved by our ethics committee. The entire coding sequence of SLC20A2, PDGFB, PDGFRB, and XPR1 was assessed by Sanger sequencing.

We included for WES 24 patients with PBC (14 probands of unrelated families and 10 apparently sporadic cases) negatively screened for all four genes.

\section{Whole-exome sequencing}

Exomes were captured using the Agilent Sureselect All Exons Human V5 Kit (Agilent technologies, Santa Clara, CA, USA). Final libraries were sequenced on a HiSeq2000 with paired ends, 100-bp reads. Reads were mapped to the 1000 Genomes GRCh37 build using BWA 0.7.5a. ${ }^{10}$ Picard Tools 1.101 was used to flag duplicate reads. We applied GATK for indel realignment, base quality score recalibration and SNPs, and indels discovery using the Haplotype Caller across all samples simultaneously according to GATK 3.3 Best Practices recommendations. ${ }^{11}$ The joint variant calling file was annotated using Annovar (date of version used: 2015/03/22 (http://www.openbioinformatics.org/annovar/).

\section{CNV calling}

To detect CNVs from WES data, we used the CANOES suite. ${ }^{12}$ Its algorithms are dedicated to the detection of CNVs using read-depth comparisons between different samples. In a first step, we calculated the read depth for each target using bedtools http://bedtools.readthedocs.io/en/latest/content/bedtools-suite. $\mathrm{html})$. We merged close targets $(<30 \mathrm{bp})$ to reduce the rate of false positives and removed all events localized in known regions of segmental duplication. As a second step, we used the genotype CNVs function of CANOES, which allows determining the probability of a specific event. The objective was to analyze each target intersecting any exon of $S L C 20 A 2, P D G F B, P D G F R B$, and XPR1 by considering each target as a putative independent event.

For exon numbering, we considered as exon 1 , the first one from $5^{\prime}$ to $3^{\prime}$ in the cDNA following the given transcript. The three SLC20A2 partial deletions were submitted to the LOVD database (http://databases.lovd.nl/shared/genes/SLC20A2/) with following variant IDs: 0000089490, 0000089491, and 0000089492.

\section{SLC20A2 QMPSF assay}

We designed a quantitative multiplex PCR of short fluorescent fragments (QMPSF) assay to confirm the presence of the deletion encompassing exon 2 of SLC2OA2 in two unrelated individuals and to screen all other patients. This assay included one control amplicon in HMBS and one amplicon in each exon of SLC20A2 (NM_006749.4) plus one amplicon in alternative (noncoding) exon 1 (NM_001257180.1), which maps between non-coding exon 1 and (coding) exon 2 of transcript NM_006749.4 in the genome. Primers are available in Supplementary Table 1.

\section{RESULTS}

Among the 24 WES of patients with PBC, mean depth of coverage was $135 \mathrm{x}$ (a mean of $98.97 \%$ of targeted bases were covered more than $15 \mathrm{x})$. We extracted all variants mapping to SLC20A2, PDGFB, $P D G F R B$, and XPR1 genes, and confirmed that no single nucleotide variant or short insertion or deletion probably affecting function had been missed by Sanger sequencing.

After exome-wide CNV calling using the CANOES tool, we detected a total of 273 events without any filtration on genes (11.4 per individual on average (range: 6-26), with a mean estimated size of $50.5 \mathrm{~kb}$ (range $0.099 \mathrm{~kb}-796.27 \mathrm{~kb}$ ); 96.7\% overlapped known CNVs from the database of genomic variants (http://dgv.tcag.ca/dgv/app/ home). In total we identified 120 duplications and 153 deletions. Of these, 110 duplications and 134 deletions were identified only once in a single patient, that is, unique CNVs. We then focused on PBC genes and identified a heterozygous deletion of exon 2 of SLC2OA2 in two unrelated probands (ROU 1129 and EXT 383, Figures 1a and b, respectively). We confirmed the presence of both deletions by QMPSF and showed that this deletion only encompassed exon 2 and not exon 1 (NM_006749.4)), alternative exon 1 (NM_001257180.1), or exon 3 . In family 1129 , the presence of the deletion was confirmed in an affected brother and his affected daughter. DNA was neither available for other affected relatives from this family nor for relatives from family EXT 383. ROU 1129 and EXT 383 families both originate from the same region of France. However, pedigree information does not suggest that they are related and we confirmed the absence of close relatedness by estimating identity by descent through Plink software (Supplementary Note 1). Clinical and imaging findings are summarized in Table 1. A more detailed description of the phenotype is available in Supplementary Note 2.

The sensitivity of CNV detection has been estimated to be $74 \%$ in the first report of CANOES using WES data obtained with another capture kit. ${ }^{12}$ With the aim to assess the presence or absence of $\mathrm{CNV}$ in each SLC20A2 exon, we developed a QMPSF assay including one amplicon in each exon of SLC20A2. After assessment of the 22 remaining patients, we detected two new heterozygous deletions: one encompassing exon 4 only (EXT 434, Figure 1c) and the other one encompassing exons 4 and 5 (EXT 451, Figure 1d). The deletion of exon 4 was also found in the the proband's affected sister. DNA from relatives of the patient carrying the exons $4-5$ deletion was not available. RNA from these patients was not available. None of the three deletions is reported in the database of genomic variants.

As 2 of the 4 deletions were missed by the first CNV screening of CANOES, we went back to WES data. We used another tool from the CANOES suite, called GenotypeCNVs, which is designed to perform targeted detection of CNVs, to test whether it allowed identifying all four deletions. The GenotypeCNVs tool allowed detecting all four deletions only after decreasing quality thresholds. However, this led to the detection of a false-positive duplication of exon 4 of SLC20A2.

\section{DISCUSSION}

SLC20A2 is the major gene causing PBC. ${ }^{13}$ It is clearly demonstrated that heterozygous loss of $S L C 20 A 2$ function is sufficient to cause PBC. ${ }^{2}$ However, only one genomic deletion has been published to date. ${ }^{7}$ Among the seven genes deleted in this family, the THAP1 gene also retained the attention as loss-of-function variants of this gene cause dystonia, which was a prominent feature associated with $\mathrm{PBC}$ in this family. We report here the first SLC20A2 partial deletions causing PBC. Among 24 patients with genetically unexplained $\mathrm{PBC}$, we found four $(16.7 \%)$ SLC20A2 partial deletions, suggesting that such events should be primarily assessed, together with SLC20A2 coding sequence variants, during the genetic screening of $\mathrm{PBC}$ patients. In our series, SLC20A2 causative variants, including sequence variants and CNVs, are found in $28.5 \%$ of unrelated probands $(40 \%$ when focusing on familial cases).

Interestingly, almost all patients with a partial SLC20A2 deletion exhibited the same radiological phenotype as patients carrying other loss-of-function variants, that is, calcification of lenticular, caudate nuclei, thalami, cerebellar hemispheres, and in particular the vermis and cortical sulci (Table 1,Supplementary Figure 1). All were symptomatic, which seems different from the $58 \%$ of symptomatic mutation carriers in our previous report. ${ }^{1}$ Of note, in family ROU-1129, the grandmother developed cognitive decline only after the age of 86 (of unknown cause), and 2 affected relatives were asymptomatic by family interview. This suggests that the apparent high proportion of symptomatic patients in the present report could be an inclusion bias.

Exon 2 of SLC2OA2 is the first coding exon and contains the translation initiation ATG codon as well as very conserved amino acid residues. This deletion probably results in a loss of function. The deletion of exon 4 causes a frameshift and therefore a probable loss of function. A splice variant at the acceptor site of intron 4 has already 

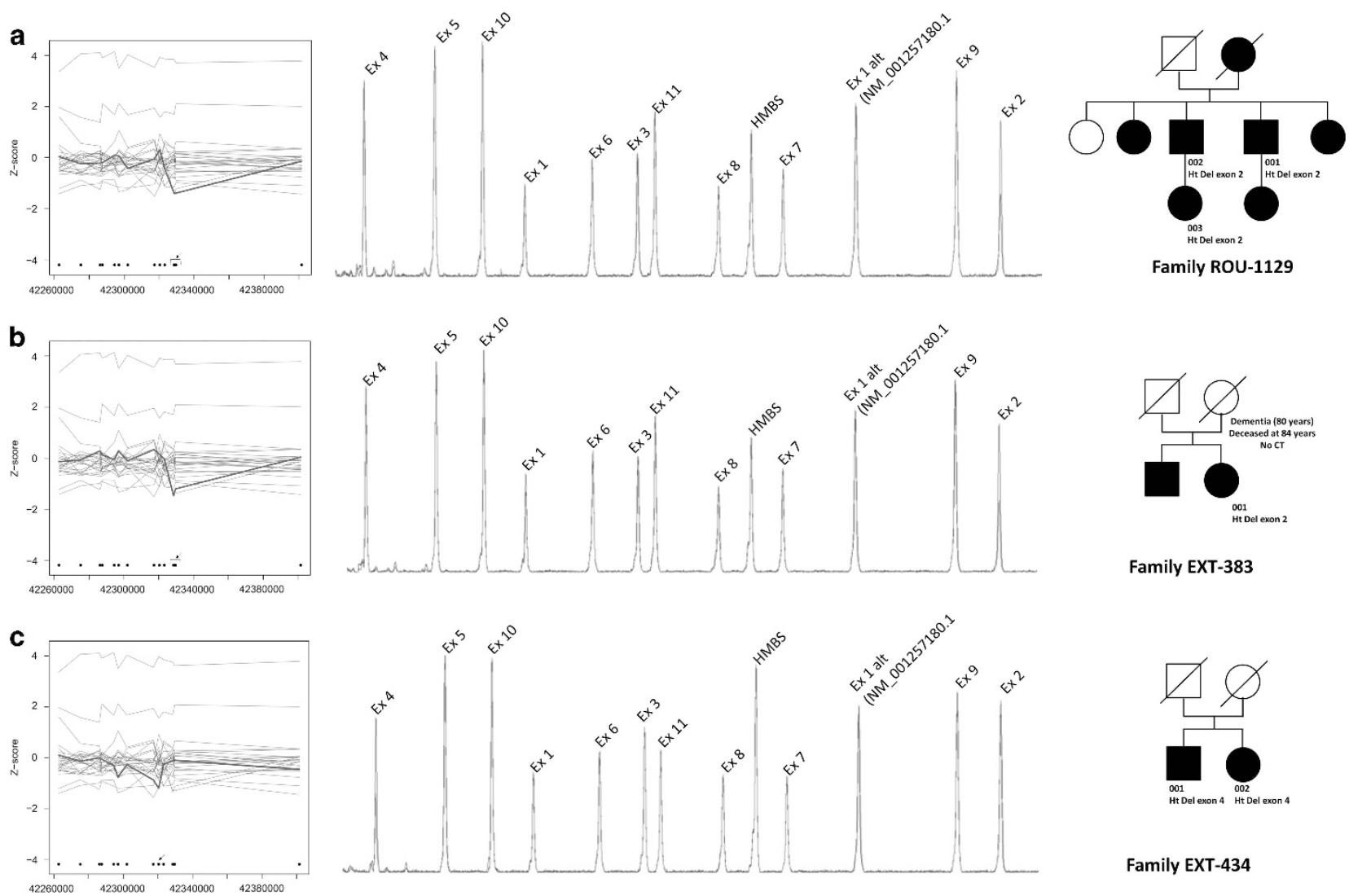

Family EXT-383
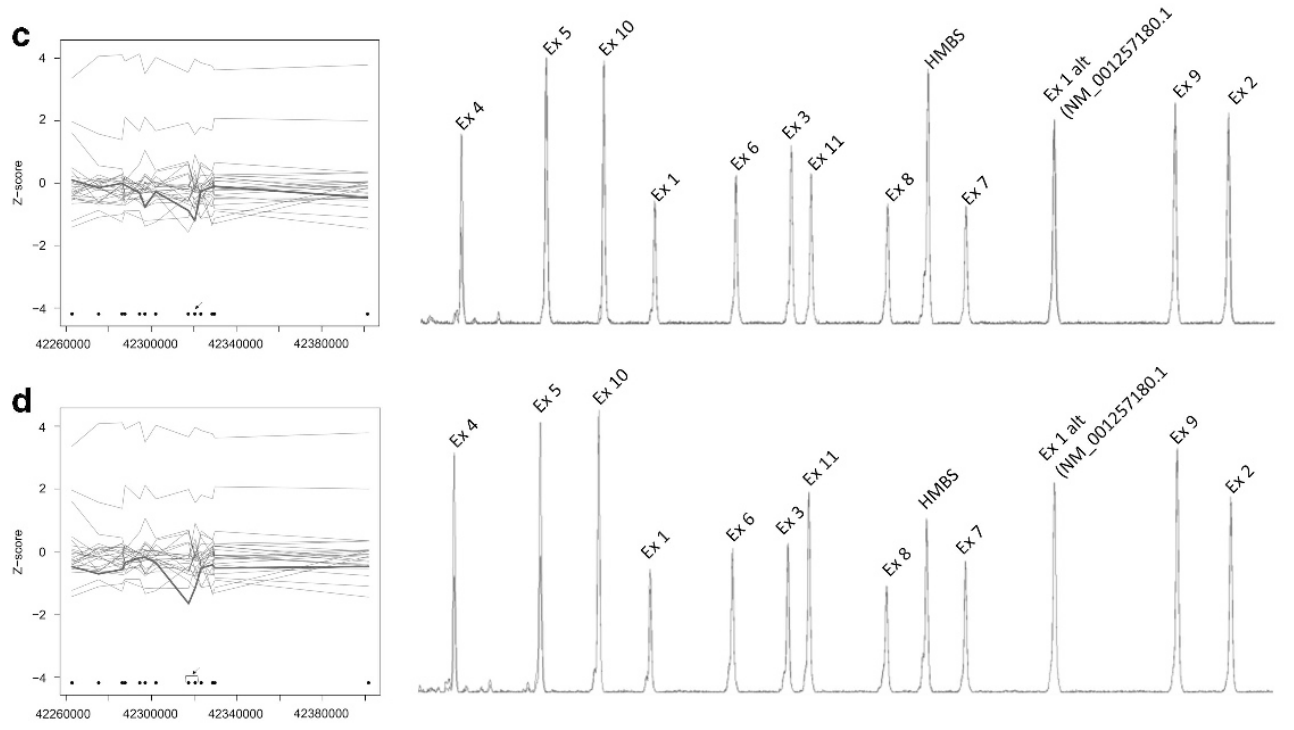

Family EXT-434
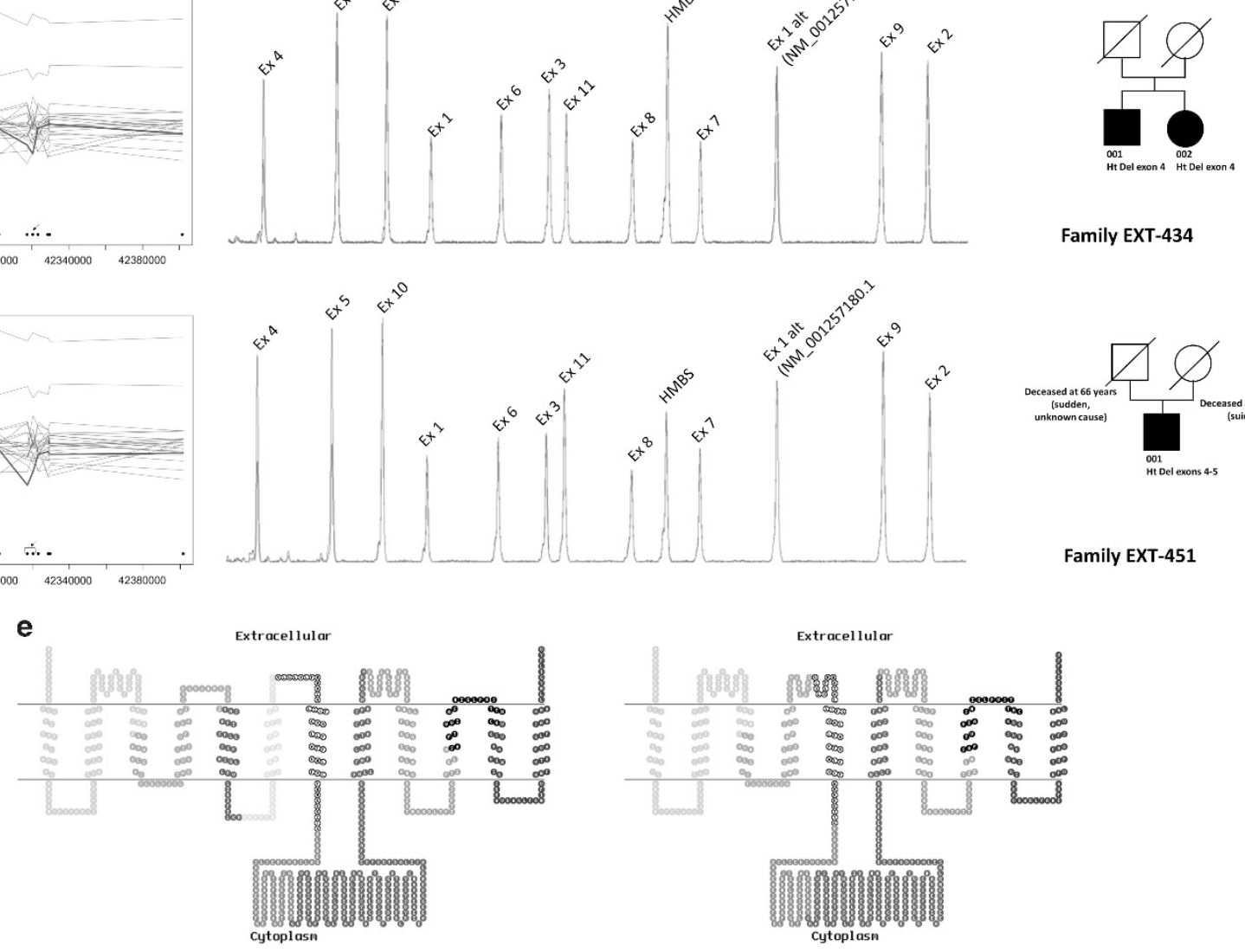

Figure 1 Partial deletions of SLC20A2. (a-d) On the left part of the panels, Z score of read-depth comparison among the 24 exomes are represented along genomic positions on chromosome 8. Red lines represent each patient carrying a partial deletion. Black spots represent the targets of the capture kit. Arrows point the targets of exon 2 (a and b), exon 4 (c), and exons 4 and 5 (d). QMPSF electropherograms of the patients show partial deletions of SLC2OA2; the patient electropherogram being in red, aligned to one control, in blue (middle part). The corresponding pedigrees are represented on the right part. (e) Schematic representation of the SLC2OA2 protein as performed by the TOPO2 online software (http://www.sacs.ucsf.edu/TOPO2/) and according to ref. 14. Each color represents one different exon, the first coding exon being exon 2 (green). Monoexonic deletions of exons 2 or 4 are not represented as they are predicted to result in no protein or a frameshift; deletion of exons 4 and 5 is represented in the right part of the panel. A full color version of this figure is available at the European Journal of Human Genetics journal online.

been described in a French family ${ }^{3}$ and could result in the same consequences as the genomic deletion of exon 4 , that is, a total skipping of exon 4 in mRNA. However, RNA was not studied in the family with the splice variant and we cannot exclude that a cryptic acceptor site could be used. The deletion of exons $4-5$ is in frame and results in the loss of two transmembrane domains (Figure 1e). Exon 4 is highly 


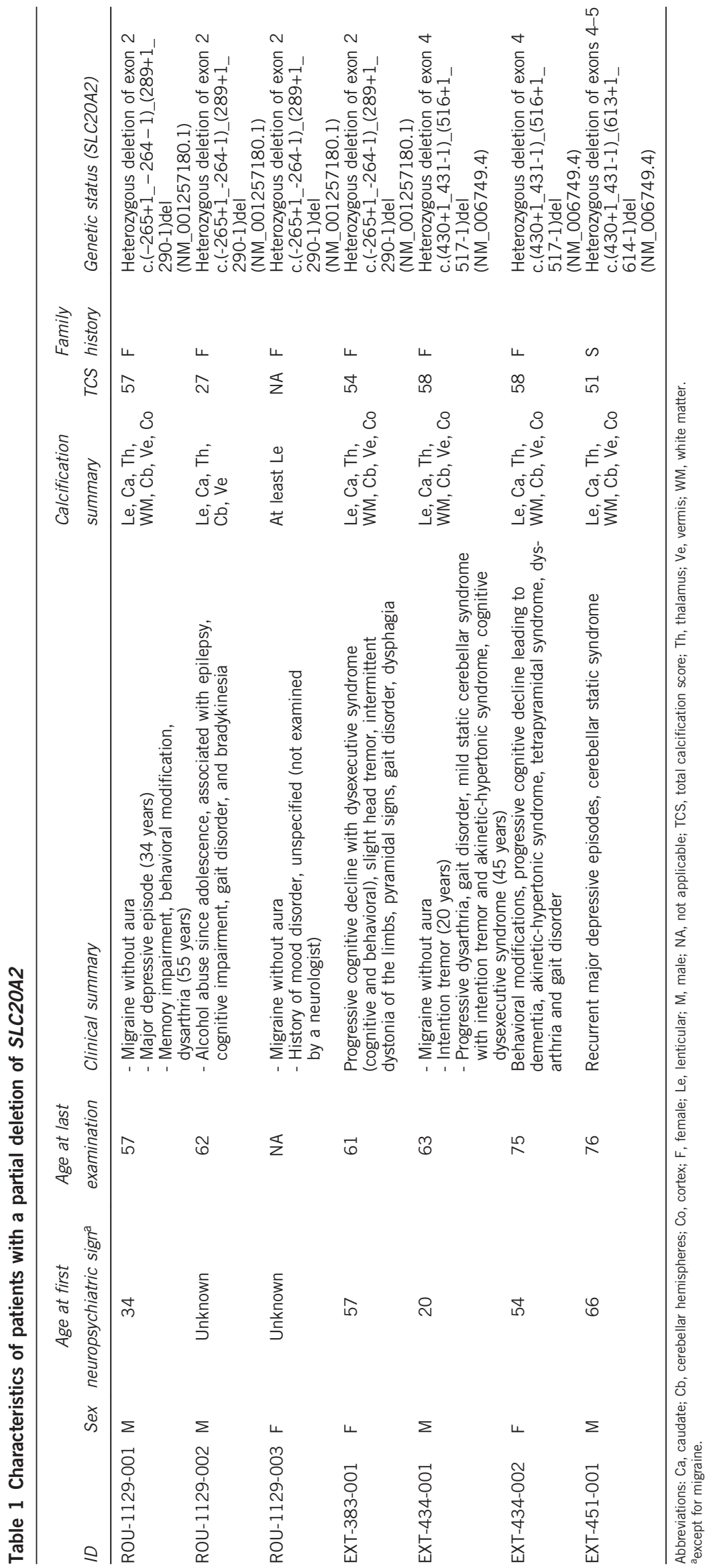


conserved during evolution and it has been shown that an artificial deletion encompassing exon 5 and other exons resulted in decreased transport activity. ${ }^{14}$ Taken together, this suggests that this deletion might also result in a loss of SLC20A2 function.

Although WES was not developed with the primary aim to detect CNVs, several tools take advantage from multiple comparisons of depth of coverage allowing the detection of deletions as short as $300 \mathrm{bp}$ (eg, the deletions involving exon 2 of SLC20A2; size of the target in the capture kit: $316 \mathrm{bp}$ ). Exon 4 of SLC20A2 is only $85 \mathrm{bp}$ long (size of the target in the capture kit: $175 \mathrm{bp}$ ) and was detected by CANOES from WES data only after targeted genotyping. This suggests that the first step of CANOES, performed exomewide, might be associated with good specificity but false negatives could be misleading. In presence of a reduced number of candidate genes, the genotyping step used with lower quality thresholds should allow reducing false negatives with the disadvantage of detecting false positives. Sensitivity and specificity of bioinformatics tools aiming at calling CNVs from WES data remain to be determined in comparison with other techniques allowing such a high resolution.

\section{CONFLICT OF INTEREST}

The authors declare no conflict of interest.

\section{ACKNOWLEDGEMENTS}

We are grateful to Camille Charbonnier for her help in relatedness estimation, and to patients and physicians involved in medical care and follow-up of patients: Philippe Baize, Nolwen Delarue, Alexandra Durr, Patrick Louf, Yann Nadjar, Gilles Olivier, Stéphane Schaeffer, and Frédéric Sedel. This study was funded by Conseil Régional de Haute Normandie - APERC 2014 no. 2014-19 in the context of Appel d'Offres Jeunes Chercheurs (CHU de Rouen). SD thanks
Fondation pour la Recherche Médicale (DEA20140630628) and JF thanks FACEPE (IBPG-0627-2.02/11; AMD-0047-2.00/15) for financial support.

1 Nicolas G, Charbonnier C, de Lemos RR et al: Brain calcification process and phenotypes according to age and sex: Lessons from SLC20A2, PDGFB, and PDGFRB mutation carriers. Am J Med Genet B Neuropsychiatr Genet 2015; 168: 586-594.

2 Wang C, Li Y, Shi L et al: Mutations in SLC20A2 link familial idiopathic basal ganglia calcification with phosphate homeostasis. Nat Genet 2012; 44: 254-256.

3 Nicolas G, Pottier C, Maltete D et al: Mutation of the PDGFRB gene as a cause of idiopathic basal ganglia calcification. Neurology 2013; 80: 181-187.

4 Keller A, Westenberger A, Sobrido MJ et al: PDGFB mutations cause brain calcifications in humans and mice. Nat Genet 2013; 45: 1077-1082.

5 Legati A, Giovannini D, Nicolas G et al: Mutations in XPR1 cause primary familial brain calcification associated with altered phosphate export. Nat Genet 2015; 47: 579-581.

6 Nicolas G, Rovelet-Lecrux A, Pottier C et al: PDGFB partial deletion: a new, rare mechanism causing brain calcification with leukoencephalopathy. J Mol Neurosci 2014; 53: 171-175.

7 Baker M, Strongosky AJ, Sanchez-Contreras MY et al: SLC2OA2 and THAP1 deletion in familial basal ganglia calcification with dystonia. Neurogenetics 2013; 15: 23-30.

8 Vanlandewijck M, Lebouvier T, Andaloussi Mae M et al: Functional characterization of germline mutations in PDGFB and PDGFRB in primary familial brain calcification. PLoS One 2015; 10: e0143407.

9 Nicolas G, Pottier C, Charbonnier C et al: Phenotypic spectrum of probable and geneticallyconfirmed idiopathic basal ganglia calcification. Brain 2013; 136: 3395-3407.

$10 \mathrm{Li} \mathrm{H}$, Durbin R: Fast and accurate long-read alignment with Burrows-Wheeler transform. Bioinformatics 2010; 26: 589-595.

11 McKenna A, Hanna M, Banks E et al: The Genome Analysis Toolkit: a MapReduce framework for analyzing next-generation DNA sequencing data. Genome Res 2010; 20: 1297-1303.

12 Backenroth D, Homsy J, Murillo LR et al: CANOES: detecting rare copy number variants from whole exome sequencing data. Nucleic Acids Res 2014; 42: e97.

13 Hsu SC, Sears RL, Lemos RR et al: Mutations in SLC20A2 are a major cause of familial idiopathic basal ganglia calcification. Neurogenetics 2013; 14: 11-22.

14 Bottger P, Pedersen L: Mapping of the minimal inorganic phosphate transporting unit of human PiT2 suggests a structure universal to PiT-related proteins from all kingdoms of life. BMC Biochem 2011; 12: 21

Supplementary Information accompanies this paper on European Journal of Human Genetics website (http://www.nature.com/ejhg) 